\title{
Effect of Variable Properties and Moving Heat Source on Magnetothermoelastic Problem under Fractional Order Thermoelasticity
}

\author{
Chunbao Xiong and Ying Guo \\ School of Civil Engineering, Tianjin University, Tianjin 300072, China \\ Correspondence should be addressed to Ying Guo; gytha_ying@tju.edu.cn
}

Received 28 January 2016; Accepted 3 May 2016

Academic Editor: Peter Majewski

Copyright (c) 2016 C. Xiong and Y. Guo. This is an open access article distributed under the Creative Commons Attribution License, which permits unrestricted use, distribution, and reproduction in any medium, provided the original work is properly cited.

A one-dimensional generalized magnetothermoelastic problem of a thermoelastic rod with finite length is investigated in the context of the fractional order thermoelasticity. The rod with variable properties, which are temperature-dependent, is fixed at both ends and placed in an initial magnetic field, and the rod is subjected to a moving heat source along the axial direction. The governing equations of the problem in the fractional order thermoelasticity are formulated and solved by means of Laplace transform in tandem with its numerical inversion. The distributions of the nondimensional temperature, displacement, and stress in the rod are obtained and illustrated graphically. The effects of the temperature-dependent properties, the velocity of the moving heat source, the fractional order parameter, and so forth on the considered variables are concerned and discussed in detail, and the results show that they significantly influence the variations of the considered variables.

\section{Introduction}

The classical coupled thermoelasticity proposed by Biot [1] predicts an infinite speed for heat propagation, which is physically impossible. To eliminate such an inherent paradox, generalized thermoelastic theories such as Lord and Shulman's theory (L-S) [2] and Green and Lindsay's theory (GL) [3] were developed in response. The L-S theory was the first description of generalized thermoelasticity in which a wave-type heat conduction law was postulated to replace the classical Fourier's law; this wave-type law is the same as that suggested by Catteneo [4] and Vernotte [5]. On the basis of these generalized models, many previous researchers have attempted to accurately capture thermomechanical behavior [6-11].

The generalized electromagnetothermoelastic problem in thermoelastic solids has attracted considerable research attention due to its extensive potential applications in diverse fields. Examples include understanding the effects of Earth's magnetic field on seismic waves, the damping of acoustic waves in magnetic fields, and the emissions of electromagnetic radiation from nuclear devices. He and Cao [12] investigated the magnetothermoelastic problem of a thin slim strip placed in a magnetic field and subjected to a moving plane of heat source. Sherief and Khader [13] studied wave propagation for a problem of an infinitely long, solid conducting circular cylinder with a traction-free lateral surface subjected to known surrounding temperatures in the presence of a uniform magnetic field in the axis direction. Abbas and Zenkour [14] presented the electromagnetothermoelastic analysis problem of an infinite functionally graded material hollow cylinder. Sarkar [15] investigated an electromagnetothermoelastic coupled problem for a half-space subjected to a thermal shock under different generalized thermoelastic theories. Pal et al. [16] dealt with the problem of magnetothermoelastic interactions in a rotating medium due to a periodically varying heat source in the context of generalized thermoelasticity. Singh and Chakraborty [17] studied the effects of the magnetic field and initial stress on the reflection of a plane magnetothermoelastic wave from the 
boundary of a solid half-space. Said [18] solved a generalized magnetothermoelastic problem for a half-space with G-N theory.

Fractional calculus has been used successfully to modify many existing models of physical processes, especially in the field of heat conduction, diffusion, viscoelasticity, solids mechanics, control theory, and electricity [19-22]. Povstenko [23] proposed a quasistatic uncoupled theory of thermoelasticity which is based on the heat conduction equation with a time-fractional order derivative. In 2010, Youssef [24] introduced the Riemann-Liouville fractional integral operator into the generalized heat conduction and constructed the theory of fractional order generalized thermoelasticity. By employing this theory, Sarkar and Lahiri [25] concerned a two-dimensional generalized thermoelastic problem for a rotating elastic medium. Youssef [26] dealt with a twotemperature generalized thermoelastic medium subjected to a moving heat source in the context of fractional order generalized thermoelasticity. Yu et al. [27] formulated the fractional order generalized electromagnetothermoelastic theory and presented the effect of fractional order parameter. Youssef and Abbas [28] solved a one-dimensional problem of an elastic half-space in the context of fractional order generalized thermoelasticity. Song et al. [29] studied the vibration of microcantilevers during a photothermal process by coupling the theories of fractional order heat conduction and elastic waves. Abbas and Youssef [30] investigated a twodimensional problem of a porous half-space with a tractionfree surface and a constant heat flux with fractional order generalized thermoelasticity theory. Recently, a completely new fractional order generalized thermoelasticity theory was introduced by Sherief et al. [31]. Based on this theory, Kothari and Mukhopadhyay [32] solved an elastic half-space problem via Laplace transform and state-space method. Sherief and Abd El-Latief [33] investigated a half-space problem with varying extent of thermal conductivity. $\mathrm{He}$ and Guo [34] investigated a one-dimensional problem for a rod subjected to a moving heat source. Sherief and Abd El-Latief [35] investigated a one-dimensional thermal shock problem for a half-space in the context of the fractional order theory of thermoelasticity and later solved a two-dimensional problem for a traction-free half-space surface subjected to a heating in the context of the same theory [36]. Abbas [37] solved the problem of fractional order thermoelastic interaction for a material placed in a magnetic field and subjected to a moving plane of heat source. Sherief and Abd El-Latief [38] solved a one-dimensional problem with a spherical cavity subjected to a thermal shock with fractional order theory of thermoelasticity. $\mathrm{Ma}$ and $\mathrm{He}$ [39] dealt with a generalized piezoelectric-thermoelastic problem subjected to a moving heat source in the context of the fractional order theory of thermoelasticity.

To explore the effects of temperature-dependent properties on predicting the dynamic behavior of problems under generalized thermoelastic theories, Ezzat et al. [40] investigated a problem in which modulus of elasticity was dependent on temperature. Allam et al. [41] studied the electromagnetothermoelastic interactions in an infinite perfectly conducting body with a spherical cavity with G-N theory.
Xiong and Tian [42] investigated the magnetothermoelastic problem of a semi-infinite body with voids and temperaturedependent material properties placed in a transverse magnetic field. Abouelregal [43] solved a one-dimensional boundary value problem of a semi-infinite piezoelectric medium with temperature-dependent properties under the theory of fractional order. The problem of the generalized thermoelastic medium for three different theories under the effects of a gravitational field was investigated by Othman et al. [44]. Pal et al. [45] dealt with a thermoelastic problem of a cylindrical cavity subjected to time-dependent thermal and mechanical shocks in the context of fractional order generalized thermoelasticity with the L-S model and G-N model. The generalized magnetothermoelastic problem of an infinite homogeneous isotropic microstretch half-space with temperature-dependent material properties placed in a transverse magnetic field was investigated in the context of different generalized thermoelastic theories by Xiong and Tian [46]. Wang et al. [47] focused on a thermoelastic problem for an elastic medium with variable properties, which they constructed in the context of the fractional order heat conduction.

To date, there are relatively few works devoted to the investigation of electromagnetothermoelastic problems involving heat source and temperature-dependent properties in the context of the fractional order theory of thermoelasticity. The present paper is devoted to our investigation of a generalized magnetothermoelastic medium with temperature-dependent properties subjected to a moving heat source in the fractional order theory, as proposed by Sherief et al. [31].

\section{Basic Equations}

The generalized magnetothermoelastic governing equations of an isotropic homogeneous conducting elastic medium under fractional order theory take the following forms:

$$
\begin{aligned}
\sigma_{i j, j}+(J \times B)_{i} & =\rho \ddot{u}_{i} \\
B & =\mu_{0} H \\
J & =\sigma_{0}(E+\dot{u} \times B) \\
\sigma_{i j} & =2 \mu e_{i j}+\left(\lambda e_{k k}-\gamma \theta\right) \delta_{i j} \\
e_{i j} & =\frac{1}{2}\left(u_{i, j}+u_{j, i}\right) \\
q_{i, i} & =-\rho T_{0} \dot{\eta}+Q \\
\rho \eta & =\gamma e_{k k}+\frac{\rho C_{E}}{T_{0}} \theta \\
\kappa_{i j} T_{i j} & =\left(1+\frac{\tau_{0}^{\alpha}}{\alpha !} \frac{\partial^{\alpha}}{\partial t^{\alpha}}\right)\left(\rho C_{E} \dot{T}+T_{0} \lambda_{i j} \dot{e}_{i j}-Q\right)
\end{aligned}
$$

$$
0<\alpha \leq 1 \text {. }
$$


The fractional derivative is defined according to Sherief et al. [31]:

$$
\frac{\partial^{\alpha}}{\partial t^{\alpha}} f(x, t)= \begin{cases}f(x, t)-f(x, 0) & \alpha \longrightarrow 0 \\ I^{1-\alpha} \frac{\partial f(x, t)}{\partial t} & 0<\alpha<1 \\ \frac{\partial f(x, t)}{\partial t} & \alpha=1,\end{cases}
$$

where the Riemann-Liouville fractional integral $I^{\alpha}$ is introduced as a natural generalization of the well-known $\alpha$-fold repeated integral $I^{\alpha} f(t)$ which is written in a convolutiontype form as follows:

$$
I^{\alpha} f(t)=\int_{0}^{t} \frac{1}{\Gamma(\alpha)}(t-s)^{\alpha-1} f(s) d s,
$$

where $\Gamma(\alpha)$ is the well-known Gamma function and $f(t)$ is Lebesgue's integrable function. Equation (8) describes the process of heat conduction in the whole spectrum. The different values of the fractional parameter within the range $0<\alpha \leq 1$ cover two cases of conductivity: $(0<\alpha<1)$ for weak conductivity and $\alpha=1$ for normal conductivity.

Here, we investigate the problem of a generalized magnetothermoelastic rod with temperature-dependent properties subjected to a moving heat source in the context of the fractional order theory of thermoelasticity. A magnetic field with constant intensity $H=\left(0, H_{0}, 0\right)$ acts perpendicularly to the axial direction of the rod, which is fixed at both ends and subjected to a moving heat source propagating along the $x$ direction. The dimension along the $x$-axis is assumed to be much greater than those along the other two directions $(y, z)$ orthogonal to the $x$-axis; thus, the problem can be treated as a one-dimensional problem.

For the one-dimensional problem, the components of the electromagnetic induction vector are given by

$$
\begin{aligned}
& B_{x}=B_{z}=0, \\
& B_{y}=\mu_{0} H_{0},
\end{aligned}
$$

while the components of Lorentz force $F=J \times B$ in the motion equation (1) can be given by

$$
\begin{aligned}
& F_{x}=-\sigma_{0} \mu_{0}^{2} H_{0}^{2}, \\
& F_{y}=F_{z}=0 .
\end{aligned}
$$

For the one-dimensional problem, the components of the displacement are

$$
\begin{aligned}
& u_{x}=u(x, t), \\
& u_{y}=u_{z}=0 .
\end{aligned}
$$

The governing equations can be simplified as follows:

$$
\begin{aligned}
& \sigma=(\lambda+2 \mu) \frac{\partial u}{\partial x}-\gamma\left(T-T_{0}\right), \\
& (\lambda+2 \mu) \frac{\partial^{2} u}{\partial x^{2}}-\gamma \frac{\partial T}{\partial x}-\sigma_{0} \mu_{0}^{2} H_{0}^{2} \frac{\partial u}{\partial t}=\rho \frac{\partial^{2} u}{\partial t^{2}}, \\
& \kappa_{i j} \frac{\partial^{2} T}{\partial x^{2}}=\left(1+\frac{\tau_{0}^{\alpha}}{\alpha !} \frac{\partial^{\alpha}}{\partial t^{\alpha}}\right)\left(\rho c_{E} \frac{\partial T}{\partial t}+\gamma T_{0} \frac{\partial^{2} u}{\partial x \partial t}-Q\right) .
\end{aligned}
$$

We consider a thermoelastic body of material with temperature-dependent properties in the following form [41]:

$$
\begin{aligned}
& \lambda=\lambda_{0} f(T), \\
& \mu=\mu_{0} f(T), \\
& \kappa=\kappa_{0} f(T), \\
& \gamma=\gamma_{0} f(T),
\end{aligned}
$$

where $\kappa$ is the thermal conductivity, $\lambda_{0}, \mu_{0}, \gamma_{0}$, and $\kappa_{0}$ are considered to be constants, and $f(T)$ is given in a nondimensional function of temperature. In the case of temperatureindependent modulus of elasticity, $f(T)=1$. We will assume the following [41]:

$$
f(T)=1-\zeta T
$$

where $\zeta$ is the empirical material constant.

In generalized thermoelasticity, as well as in the coupled theory, only the infinitesimal temperature deviations from the reference temperature are considered. For linearity of the governing partial differential equations of the problem, we have to take into account the condition $\left|T-T_{0}\right| / T_{0} \ll 1$, which gives the approximating function of $f(T)$ in the following form:

$$
f(T) \approx 1-\zeta T_{0}
$$

For convenience, the following nondimensional quantities are also introduced:

$$
\begin{aligned}
x^{*} & =c_{0} \eta_{0} x, \\
u^{*} & =c_{0} \eta_{0} u, \\
t^{*} & =c_{0}^{2} \eta_{0} t, \\
\tau_{0}{ }^{*} & =c_{0}^{2} \eta_{0} \tau_{0}, \\
\theta^{*} & =\frac{T-T_{0}}{T_{0}}, \\
\sigma^{*} & =\frac{\sigma}{\mu}, \\
Q^{*} & =\frac{Q}{k T_{0} c_{0}^{2} \eta_{0}^{2}}, \\
c_{0}^{2} & =\frac{\lambda+2 \mu}{\rho}, \\
\eta_{0} & =\frac{\rho C_{E}}{k} .
\end{aligned}
$$


In terms of the nondimensional variables in (18), (14) take the following forms (dropping the asterisks for convenience):

$$
\begin{aligned}
\vartheta \sigma & =\beta^{2} \frac{\partial u}{\partial x}-b \theta \\
\frac{\partial^{2} u}{\partial x^{2}}-\frac{b}{\beta^{2}} \frac{\partial \theta}{\partial x} & =\vartheta\left(\frac{\partial^{2} u}{\partial t^{2}}+\varepsilon \frac{\partial u}{\partial t}\right) \\
\frac{\partial^{2} \theta}{\partial x^{2}} & =\left(1+\frac{\tau_{0}^{\alpha}}{\alpha !} \frac{\partial^{\alpha}}{\partial t^{\alpha}}\right)\left(\vartheta \frac{\partial \theta}{\partial t}+g \frac{\partial^{2} u}{\partial x \partial t}-Q\right),
\end{aligned}
$$

where

$$
\begin{aligned}
\vartheta & =\frac{1}{1-\zeta T_{0}}, \\
\beta^{2} & =\frac{\lambda+2 \mu}{\mu}, \\
b & =\frac{\gamma T_{0}}{\mu}, \\
g & =\frac{\gamma}{\vartheta \rho C_{E}}, \\
\varepsilon & =\frac{\sigma_{0} \mu_{0}^{2} H_{0}^{2}}{\eta_{0}(\lambda+2 \mu)} .
\end{aligned}
$$

The rod is assumed to have reference temperature $T_{0}$ and homogeneous initial conditions:

$$
\begin{aligned}
& u(x, 0)=\dot{u}(x, 0)=0, \\
& \theta(x, 0)=\dot{\theta}(x, 0)=0 .
\end{aligned}
$$

The rod is fixed at both ends with a nondimensional length $l$, so the boundary conditions are

$$
\begin{gathered}
u(0, t)=u(l, t)=0, \\
\frac{\partial \theta(0, t)}{\partial x}=\frac{\partial \theta(l, t)}{\partial x}=0 .
\end{gathered}
$$

The heat source is moving along the $x$-axis with a constant velocity $v$, which can be described as follows:

$$
Q=Q_{0} \delta(x-v t),
$$

where $Q_{0}$ is a constant and $\delta$ is the delta function.

Applying the Laplace transform defined by

$$
L[f(t)]=\bar{f}(p)=\int_{0}^{\infty} e^{-p t} f(t) d t \quad \operatorname{Re}(p)>0
$$

to (19) with (21), we obtain

$$
\begin{aligned}
& \vartheta \bar{\sigma}=\beta^{2} \frac{d \bar{u}}{d x}-b \bar{\theta}, \\
& \frac{d^{2} \bar{u}}{d x^{2}}-\frac{b}{\beta^{2}} \frac{d \bar{\theta}}{d x}=9 p(p+\varepsilon) \bar{u}, \\
& \frac{d^{2} \bar{\theta}}{d x^{2}}=\left(1+\frac{\tau_{0}^{\alpha}}{\alpha !} p^{\alpha}\right)\left(p 9 \bar{\theta}+g p \frac{d \bar{u}}{d x}-\omega \vartheta e^{-(p / v) x}\right),
\end{aligned}
$$

where

$$
\omega=\frac{Q_{0}}{v} .
$$

By applying the Laplace transform, the boundary conditions in (21) can be expressed as follows:

$$
\begin{gathered}
\bar{u}(0, p)=\bar{u}(l, p)=0, \\
\frac{d \bar{\theta}(0, p)}{d x}=\frac{d \bar{\theta}(l, p)}{d x}=0 .
\end{gathered}
$$

\section{Solutions in the Laplace Domain}

Eliminating $\bar{\theta}$ between (26) and (27), we obtain the following equation satisfied by $\bar{u}$ :

$$
\frac{d^{4} \bar{u}}{d x^{4}}-m_{1} \frac{d^{2} \bar{u}}{d x^{2}}+m_{2} \bar{u}=m_{3} e^{-(p / v) x},
$$

where

$$
\begin{aligned}
& m_{1}=\left(\vartheta+\frac{g b}{\beta^{2}}\right)\left(1+\frac{\tau_{0}^{\alpha}}{\alpha !} p^{\alpha}\right) p+\vartheta p(p+\varepsilon), \\
& m_{2}=\left(1+\frac{\tau_{0}^{\alpha}}{\alpha !} p^{\alpha}\right) \vartheta^{2} p^{2}(p+\varepsilon), \\
& m_{3}=\frac{b \omega p \vartheta\left(1+\left(\tau_{0}^{\alpha} / \alpha !\right) p^{\alpha}\right)}{\beta^{2} v} .
\end{aligned}
$$

The general solution of (30) is

$$
\begin{aligned}
\bar{u}= & C_{1} e^{-k_{1} x}+C_{2} e^{k_{1} x}+C_{3} e^{-k_{2} x}+C_{4} e^{k_{2} x} \\
& +C_{5} e^{-(p / v) x},
\end{aligned}
$$

where $C_{i}(i=1,2,3,4)$ are parameters depending on $p$ to be determined from the boundary conditions, and

$$
C_{5}=\frac{m_{3}}{\left[(p / v)^{4}-m_{1}(p / v)^{2}+m_{2}\right]},
$$

where $k_{1}$ and $k_{2}$ are the roots of the following characteristic equation:

$$
k^{4}-m_{1} k^{2}+m_{2}=0,
$$

and $k_{1}, k_{2}$ are given by

$$
\begin{aligned}
& k_{1}=\sqrt{\frac{m_{1}+\sqrt{m_{1}^{2}-4 m_{2}}}{2}}, \\
& k_{2}=\sqrt{\frac{m_{1}-\sqrt{m_{1}^{2}-4 m_{2}}}{2}} .
\end{aligned}
$$

Similarly, eliminating $\bar{u}$ between (26) and (27), we obtain

$$
\frac{d^{4} \bar{\theta}}{d x^{4}}-m_{1} \frac{d^{2} \bar{\theta}}{d x^{2}}+m_{2} \bar{\theta}=m_{4} e^{-(p / v) x},
$$


where

$$
m_{4}=\omega \vartheta\left(1+\frac{\tau_{0}^{\alpha}}{\alpha !} p^{\alpha}\right)\left[\vartheta p(p+\varepsilon)-\frac{p^{2}}{v^{2}}\right] .
$$

The general solution of (36) is

$$
\begin{aligned}
\bar{\theta}= & C_{11} e^{-k_{1} x}+C_{22} e^{k_{1} x}+C_{33} e^{-k_{2} x}+C_{44} e^{k_{2} x} \\
& +C_{55} e^{-(p / v) x},
\end{aligned}
$$

where the parameters of $C_{i i}(i=1,2,3,4)$ are dependent on p.

Substituting $\bar{u}$ from (32) and $\bar{\theta}$ from (38) into (26), the following relationships become clear:

$$
\begin{aligned}
& C_{11}=-\frac{\beta^{2}\left[k_{1}^{2}-\vartheta p(p+\varepsilon)\right]}{b k_{1}} C_{1}, \\
& C_{22}=\frac{\beta^{2}\left[k_{1}^{2}-\vartheta p(p+\varepsilon)\right]}{b k_{1}} C_{2}, \\
& C_{33}=-\frac{\beta^{2}\left[k_{2}^{2}-\vartheta p(p+\varepsilon)\right]}{b k_{2}} C_{3}, \\
& C_{44}=\frac{\beta^{2}\left[k_{2}^{2}-\vartheta p(p+\varepsilon)\right]}{b k_{2}} C_{4}, \\
& C_{55}=\frac{\beta^{2}\left[v^{2} \vartheta(p+\varepsilon)-p\right]}{b v} C_{5} .
\end{aligned}
$$

To obtain the parameters of $C_{i}(i=1,2,3,4)$ and $C_{i i}(i=$ $1,2,3,4),(32)$ and (38) are substituted into the equation of boundary conditions as follows:

$$
\begin{aligned}
& C_{1}+C_{2}+C_{3}+C_{4}=-C_{5}, \\
& C_{1} e^{-k_{1} l}+C_{2} e^{k_{1} l}+C_{3} e^{-k_{2} l}+C_{4} e^{k_{2} l}=-C_{5} e^{-(p / v) l}, \\
& -C_{11} k_{1}+C_{22} k_{1}-C_{33} k_{2}+C_{44} k_{2}=\left(\frac{p}{v}\right) C_{55}, \\
& -C_{11} k_{1} e^{-k_{1} l}+C_{22} k_{1} e^{k_{1} l}-C_{33} k_{2} e^{-k_{2} l}+C_{44} k_{2} e^{k_{2} l} \\
& \quad=\left(\frac{p}{v}\right) C_{55} e^{-(p / v) l} .
\end{aligned}
$$

Solving (40), we obtain $C_{i}(i=1,2,3,4)$ as follows:

$$
\begin{aligned}
& C_{1}=\frac{\left(k_{2}^{2}-p^{2} / v^{2}\right)\left(e^{k_{1} l}-e^{-(p / v) l}\right)}{\left(k_{1}^{2}-k_{2}^{2}\right)\left(e^{k_{1} l}-e^{-k_{1} l}\right)} C_{5}, \\
& C_{2}=-\frac{\left(k_{2}^{2}-p^{2} / v^{2}\right)\left(e^{-k_{1} l}-e^{-(p / v) l}\right)}{\left(k_{1}^{2}-k_{2}^{2}\right)\left(e^{k_{1} l}-e^{-k_{1} l}\right)} C_{5}, \\
& C_{3}=-\frac{\left(k_{1}^{2}-p^{2} / v^{2}\right)\left(e^{k_{2} l}-e^{-(p / v) l}\right)}{\left(k_{1}^{2}-k_{2}^{2}\right)\left(e^{k_{2} l}-e^{-k_{2} l}\right)} C_{5}, \\
& C_{4}=\frac{\left(k_{1}^{2}-p^{2} / v^{2}\right)\left(e^{-k_{2} l}-e^{-(p / v) l}\right)}{\left(k_{1}^{2}-k_{2}^{2}\right)\left(e^{k_{2} l}-e^{-k_{2} l}\right)} C_{5} .
\end{aligned}
$$

Substituting (41) into (32), we obtain

$$
\begin{aligned}
\bar{u}= & \frac{\left(k_{2}^{2}-p^{2} / v^{2}\right)\left(e^{k_{1} l}-e^{-(p / v) l}\right)}{\left(k_{1}^{2}-k_{2}^{2}\right)\left(e^{k_{1} l}-e^{-k_{1} l}\right)} C_{5} e^{-k_{1} x} \\
& -\frac{\left(k_{2}^{2}-p^{2} / v^{2}\right)\left(e^{-k_{1} l}-e^{-(p / v) l}\right)}{\left(k_{1}^{2}-k_{2}^{2}\right)\left(e^{k_{1} l}-e^{-k_{1} l}\right)} C_{5} e^{k_{1} x} \\
& -\frac{\left(k_{1}^{2}-p^{2} / v^{2}\right)\left(e^{k_{2} l}-e^{-(p / v) l}\right)}{\left(k_{1}^{2}-k_{2}^{2}\right)\left(e^{k_{2} l}-e^{-k_{2} l}\right)} C_{5} e^{-k_{2} x} \\
& +\frac{\left(k_{1}^{2}-p^{2} / v^{2}\right)\left(e^{-k_{2} l}-e^{-(p / v) l}\right)}{\left(k_{1}^{2}-k_{2}^{2}\right)\left(e^{k_{2} l}-e^{-k_{2} l}\right)} C_{5} e^{k_{2} x} \\
& +C_{5} e^{-(p / v) x} .
\end{aligned}
$$

The relationship between $C_{i}$ and $C_{i i}$ in (39) gives

$$
\begin{aligned}
C_{11} & =-\frac{\beta^{2}\left[k_{1}{ }^{2}-9 p(p+\varepsilon)\right]\left(k_{2}{ }^{2}-p^{2} / v^{2}\right)\left(e^{k_{1} l}-e^{-(p / v) l}\right)}{b k_{1}\left(k_{1}{ }^{2}-k_{2}{ }^{2}\right)\left(e^{k_{1} l}-e^{-k_{1} l}\right)} \\
\cdot & C_{5}, \\
C_{22} & \\
& =-\frac{\beta^{2}\left[k_{1}{ }^{2}-\vartheta p(p+\varepsilon)\right]\left(k_{2}{ }^{2}-p^{2} / v^{2}\right)\left(e^{-k_{1} l}-e^{-(p / v) l}\right)}{b k_{1}\left(k_{1}{ }^{2}-k_{2}{ }^{2}\right)\left(e^{k_{1} l}-e^{-k_{1} l}\right)} \\
\cdot & C_{5}, \\
C_{33} & =\frac{\beta^{2}\left[k_{2}{ }^{2}-9 p(p+\varepsilon)\right]\left(k_{1}{ }^{2}-p^{2} / v^{2}\right)\left(e^{k_{2} l}-e^{-(p / v) l}\right)}{b k_{2}\left(k_{1}{ }^{2}-k_{2}{ }^{2}\right)\left(e^{k_{2} l}-e^{-k_{2} l}\right)} \\
\cdot & C_{5}, \\
C_{44} & =\frac{\beta^{2}\left[k_{2}{ }^{2}-9 p(p+\varepsilon)\right]\left(k_{1}{ }^{2}-p^{2} / v^{2}\right)\left(e^{-k_{2} l}-e^{-(p / v) l}\right)}{b k_{2}\left(k_{1}{ }^{2}-k_{2}{ }^{2}\right)\left(e^{k_{2} l}-e^{-k_{2} l}\right)} \\
\cdot & C_{5}, \\
C_{55} & =\frac{\beta^{2}\left[v^{2} \vartheta(p+\varepsilon)-p\right]}{b v} C_{5} .
\end{aligned}
$$

Substituting (43) into (38), we obtain

$$
\begin{aligned}
\bar{\theta} & =-\frac{\beta^{2}\left[k_{1}{ }^{2}-\vartheta p(p+\varepsilon)\right]\left(k_{2}{ }^{2}-p^{2} / v^{2}\right)\left(e^{k_{1} l}-e^{-(p / v) l}\right)}{b k_{1}\left(k_{1}{ }^{2}-k_{2}{ }^{2}\right)\left(e^{k_{1} l}-e^{-k_{1} l}\right)} \\
& \cdot C_{5} e^{-k_{1} x} \\
& -\frac{\beta^{2}\left[k_{1}{ }^{2}-\vartheta p(p+\varepsilon)\right]\left(k_{2}{ }^{2}-p^{2} / v^{2}\right)\left(e^{-k_{1} l}-e^{-(p / v) l}\right)}{b k_{1}\left(k_{1}{ }^{2}-k_{2}{ }^{2}\right)\left(e^{k_{1} l}-e^{-k_{1} l}\right)} \\
& \cdot C_{5} e^{k_{1} x}
\end{aligned}
$$




$$
\begin{aligned}
& +\frac{\beta^{2}\left[k_{2}{ }^{2}-\vartheta p(p+\varepsilon)\right]\left(k_{1}{ }^{2}-p^{2} / v^{2}\right)\left(e^{k_{2} l}-e^{-(p / v) l}\right)}{b k_{2}\left(k_{1}{ }^{2}-k_{2}{ }^{2}\right)\left(e^{k_{2} l}-e^{-k_{2} l}\right)} \\
& \cdot C_{5} e^{-k_{2} x} \\
& +\frac{\beta^{2}\left[k_{2}{ }^{2}-\vartheta p(p+\varepsilon)\right]\left(k_{1}{ }^{2}-p^{2} / v^{2}\right)\left(e^{-k_{2} l}-e^{-(p / v) l}\right)}{b k_{2}\left(k_{1}{ }^{2}-k_{2}{ }^{2}\right)\left(e^{k_{2} l}-e^{\left.-k_{2} l\right)}\right.} \\
& \cdot C_{5} e^{k_{2} x}+\frac{\beta^{2}\left[v^{2} \vartheta(p+\varepsilon)-p\right]}{b v} C_{5} e^{-(p / v) x} .
\end{aligned}
$$

Substituting (42) and (44) into (25) yields

$$
\begin{aligned}
\bar{\sigma}= & -\frac{\beta^{2} p(p+\varepsilon)\left(k_{2}^{2}-p^{2} / v^{2}\right)\left(e^{k_{1} l}-e^{-(p / v) l}\right)}{k_{1}\left(k_{1}^{2}-k_{2}^{2}\right)\left(e^{k_{1} l}-e^{-k_{1} l}\right)} \\
& \cdot C_{5} e^{-k_{1} x} \\
- & \frac{\beta^{2} p(p+\varepsilon)\left(k_{2}^{2}-p^{2} / v^{2}\right)\left(e^{-k_{1} l}-e^{-(p / v) l}\right)}{k_{1}\left(k_{1}^{2}-k_{2}^{2}\right)\left(e^{k_{1} l}-e^{-k_{1} l}\right)} \\
& \cdot C_{5} e^{k_{1} x} \\
+ & \frac{\beta^{2} p(p+\varepsilon)\left(k_{1}^{2}-p^{2} / v^{2}\right)\left(e^{k_{2} l}-e^{-(p / v) l}\right)}{k_{2}\left(k_{1}^{2}-k_{2}^{2}\right)\left(e^{k_{2} l}-e^{-k_{2} l}\right)} \\
& \cdot C_{5} e^{-k_{2} x} \\
+ & \frac{\beta^{2} p(p+\varepsilon)\left(k_{1}^{2}-p^{2} / v^{2}\right)\left(e^{-k_{2} l}-e^{-(p / v) l}\right)}{k_{2}\left(k_{1}^{2}-k_{2}^{2}\right)\left(e^{k_{2} l}-e^{-k_{2} l}\right)} \\
& \cdot C_{5} e^{k_{2} x}-\beta^{2} v(p+\varepsilon) C_{5} e^{-(p / v) x} .
\end{aligned}
$$

\section{Numerical Inversion of the Transforms}

In order to determine the nondimensional temperature, nondimensional displacement, and nondimensional stress in the rod, we need to invert the parameters of $\bar{\theta}, \bar{u}$, and $\bar{\sigma}$ from the Laplace domain. Unfortunately, the obtained solutions in the Laplace domain are too complicated to be inverted analytically; thus, a feasible numerical method, that is, the Riemann-sum approximation method, is used to complete the inversion. Accordingly, any function $\bar{f}(x, p)$ in the Laplace domain can be inverted to the time domain as follows [48]:

$$
\begin{aligned}
f(x, t) & \\
& =\frac{e^{\beta t}}{t}\left[\frac{1}{2} \bar{f}(x, \beta)+\operatorname{Re} \sum_{n=1}^{N} \bar{f}\left(x, \beta+\frac{i n \pi}{t}\right)(-1)^{n}\right],
\end{aligned}
$$

where $\operatorname{Re}$ is the real part and $i$ is the imaginary number unit. For faster convergence, numerous numerical experiments have shown that the value of $\beta$ satisfies the relation $\beta t \approx 4.7$ [48].

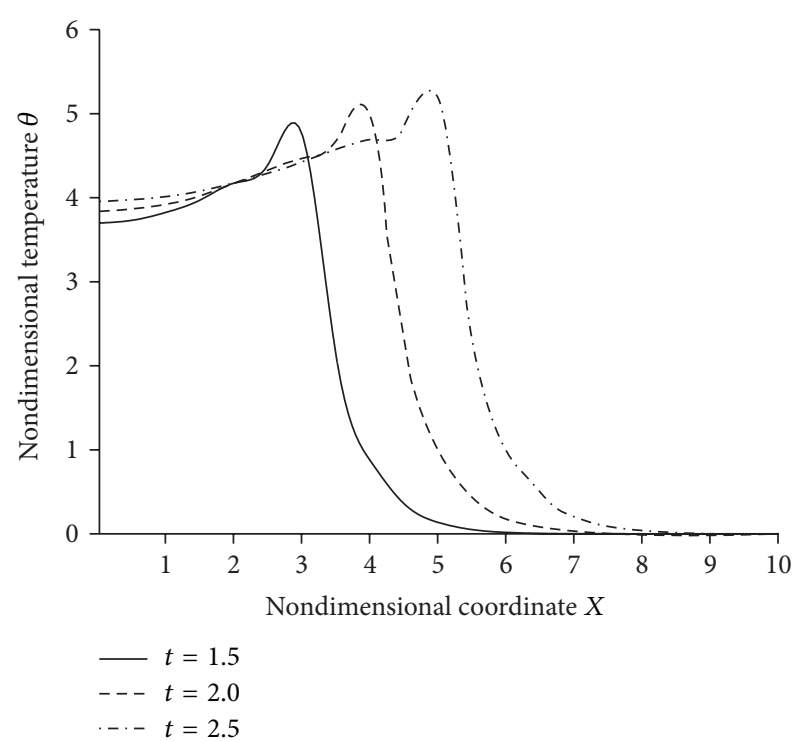

FIGURE 1: Nondimensional temperature distribution for different $t$ values.

\section{Numerical Results and Discussions}

In terms of the Riemann-sum approximation defined in (46), numerical Laplace inversion is implemented to obtain the nondimensional temperature, displacement, and stress in the rod in the time domain. For the purposes of simulation, the thermoelastic material is specified as copper and the parameters are

$$
\begin{aligned}
\lambda & =7.76 \times 10^{10} \mathrm{Nm}^{-2}, \\
\mu & =3.86 \times 10^{10} \mathrm{Nm}^{-2}, \\
\rho & =8954 \mathrm{kgm}^{-3}, \\
\alpha_{t} & =1.78 \times 10^{-5} \mathrm{~K}^{-1}, \\
C_{E} & =383.1 \mathrm{JKg}^{-1} \mathrm{~K}^{-1}, \\
\kappa & =386 \mathrm{Wm}^{-1} \mathrm{~K}^{-1}, \\
T_{0} & =293 \mathrm{~K}, \\
\zeta & =0.0005 \mathrm{~K}^{-1} .
\end{aligned}
$$

The other constants are

$$
\begin{aligned}
Q_{0} & =10, \\
\tau_{0} & =0.05, \\
l & =10 .
\end{aligned}
$$

Numerical calculation is carried out for the following five cases.

In Case 1, we investigate the nondimensional temperature, displacement, and stress varying with time as shown in Figures 1-3 with the moving heat source velocity, fractional order parameter, value of temperature-dependent properties, 


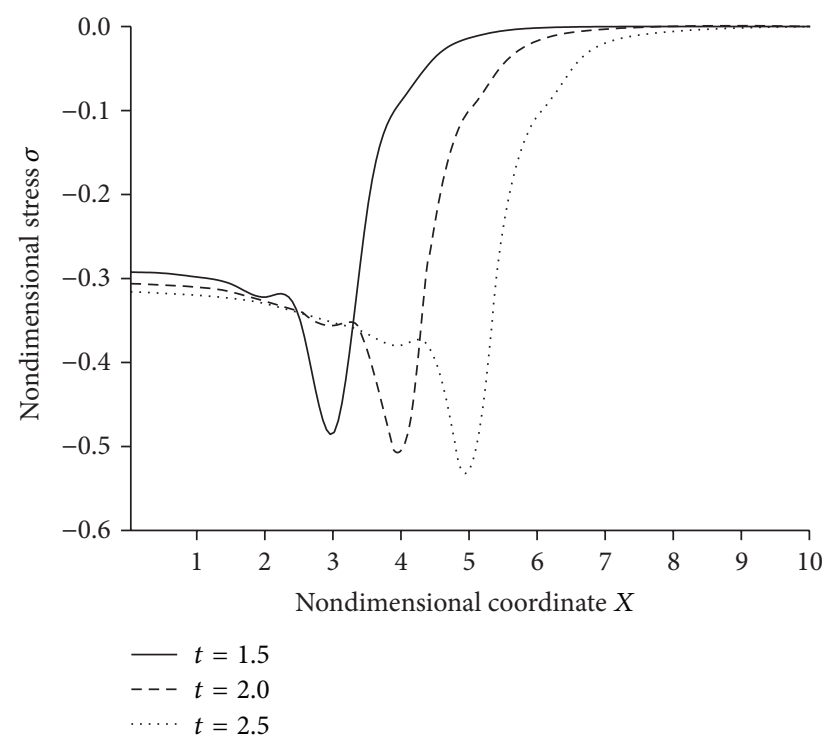

Figure 2: Nondimensional stress distribution for different $t$ values.

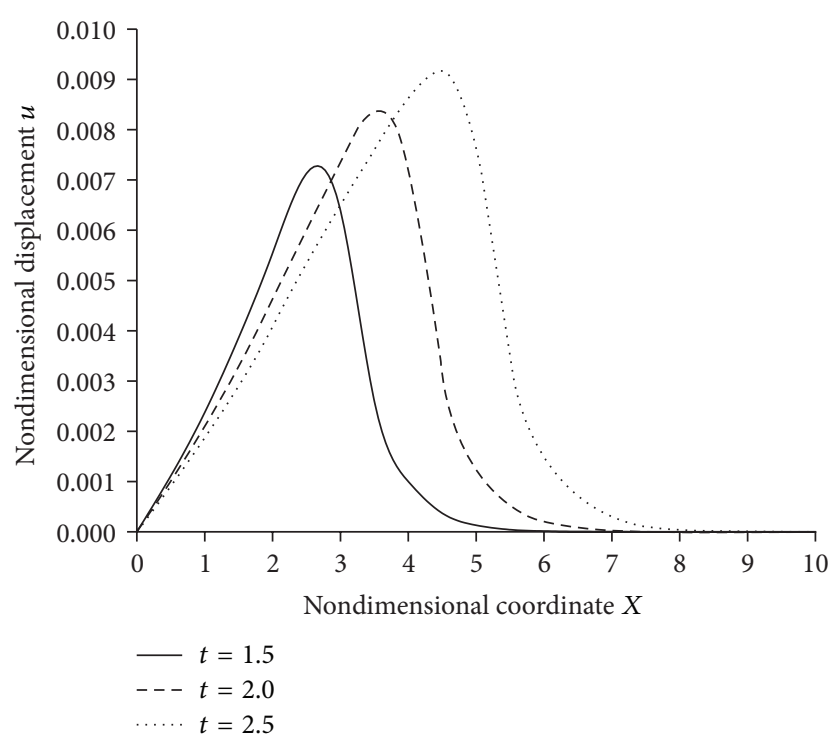

FIgURE 3: Nondimensional displacement distribution for different $t$ values.

and magnetic field set as constants (i.e., $v=2.0, \alpha=0.25$, $\vartheta=0.5, \varepsilon=5$ ). In Case 2 , the considered variables varying with different moving heat source velocity are investigated when $t=1.5, \alpha=0.25, \vartheta=0.5$, and $\varepsilon=5$ (Figures 4$6)$. In Case 3 , we investigate how the considered variables vary with different magnetic fields when $t=1.5, v=2.0$, $\vartheta=0.5$, and $\alpha=0.25$ (Figures 7-9). Case 4 involves the considered variables varying with different temperaturedependent properties when $t=1.5, v=2.0, \alpha=0.25$, and $\varepsilon=5$ (Figures 10-12), and, in Case 5, we investigate how the considered variables vary with different fractional order parameters when $t=1.5, v=2.0, \vartheta=0.5$, and, $\varepsilon=5$ (Figures 13-15).

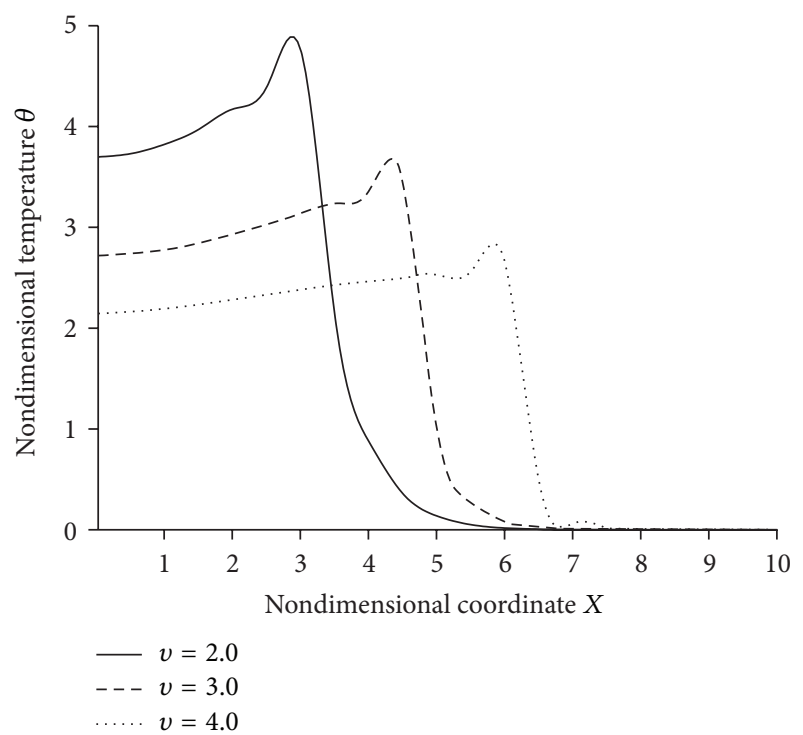

FIGURE 4: Nondimensional temperature distribution for different $v$ values.

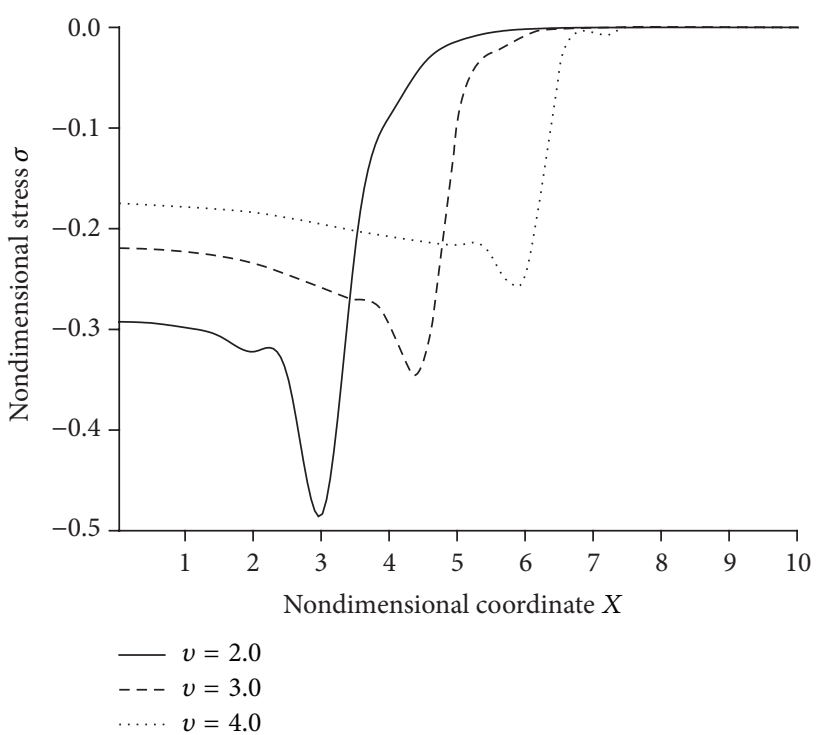

FIGURE 5: Nondimensional stress distribution for different $v$ values.

In Figures 1-3 (Case 1), the solid line, dash line, and dot line refer to $t=1.5, t=2.0$, and $t=2.5$, respectively. Figure 1 shows that the nondimensional temperature increases as the time increases. At location $x=v t$, the heat source releases its maximum energy which leads to a peak value. As shown in Figure 2, the nondimensional stress in the rod is compressive. Due to the fixed ends, thermal expansion deformation is restrained in both ends and leads to the occurrence of compressive thermal stress in the rod. The absolute value of stress increases with the passage of time. As shown in Figure 3, the nondimensional displacement also increases as time progresses. Due to the applied moving heat source, the rod undergoes thermal expansion deformation. With the passage of time, the heat disturbed region enlarges so that thermal expansion deformation evolves along the rod. 


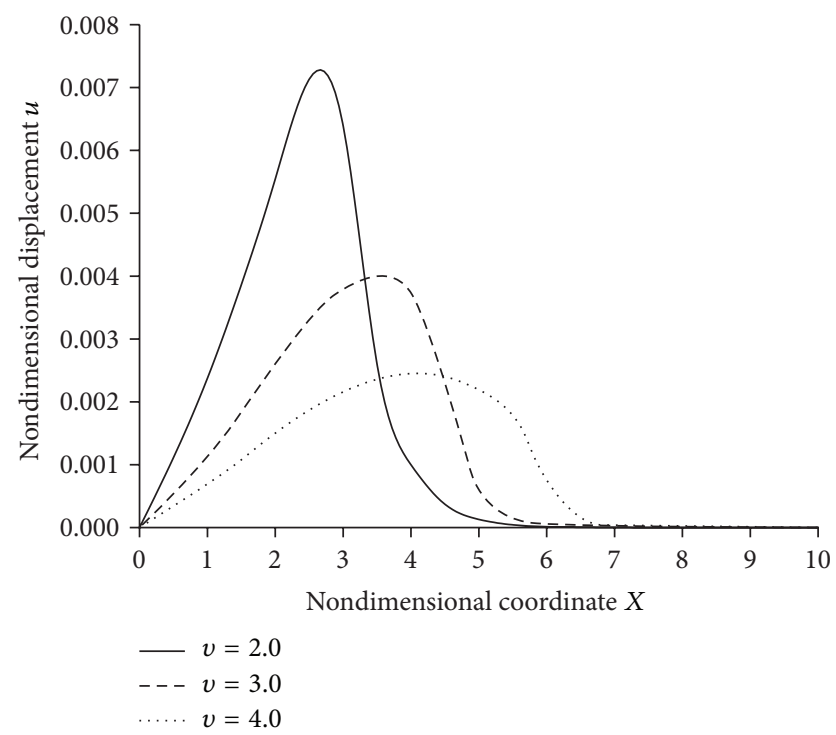

FIGURE 6: Nondimensional displacement distribution for different $v$ values.

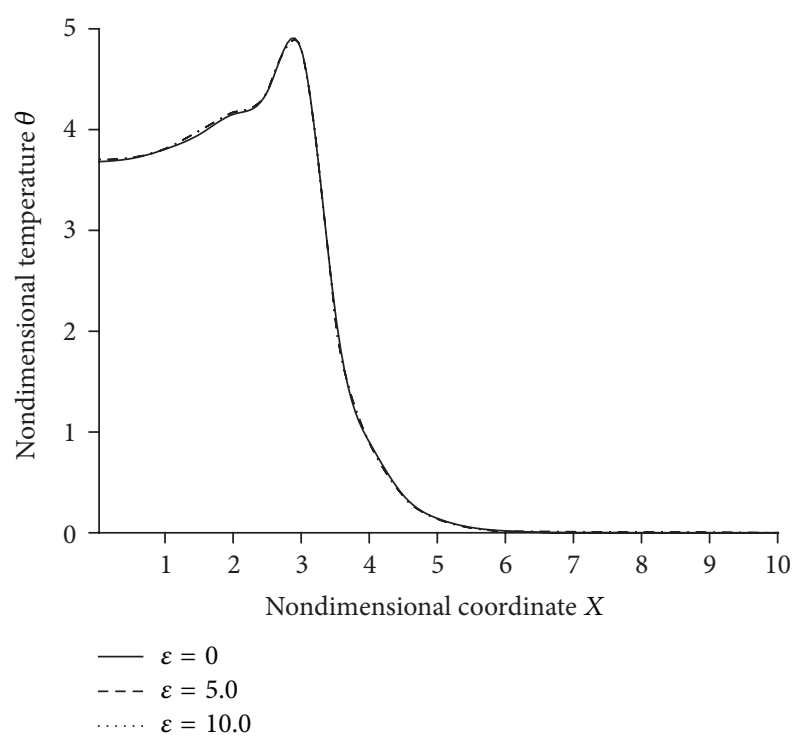

FIGURE 7: Nondimensional temperature distribution for different $\varepsilon$ values.

In Figures 4-6 (Case 2), the solid line, dash line, and dot line refer to $v=2.0, v=3.0$, and $v=4.0$, respectively. As shown in Figure 4, the nondimensional temperature decreases with the increasing of the moving heat resource velocity. In a given period, the energy that the heat source can release is constant; however, the intensity of the released energy per unit length decreases as the source speed increases. It is also clear in Figures 5 and 6 that the magnitudes of the nondimensional stress and displacement decrease as the moving heat source velocity increases, which is the result of reduction in heat energy intensity per unit length at greater velocities.

In Figures 7-9 (Case 3), the solid line, dash line, and dot line refer to $\varepsilon=0, \varepsilon=5.0$, and $\varepsilon=10.0$, respectively.

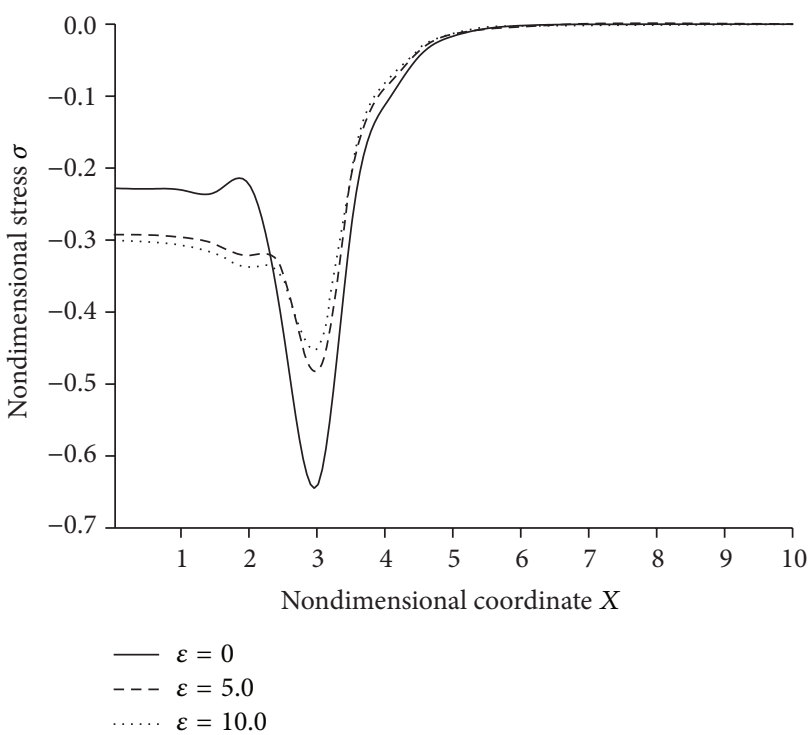

FIGURE 8: Nondimensional stress distribution for different $\varepsilon$ values.

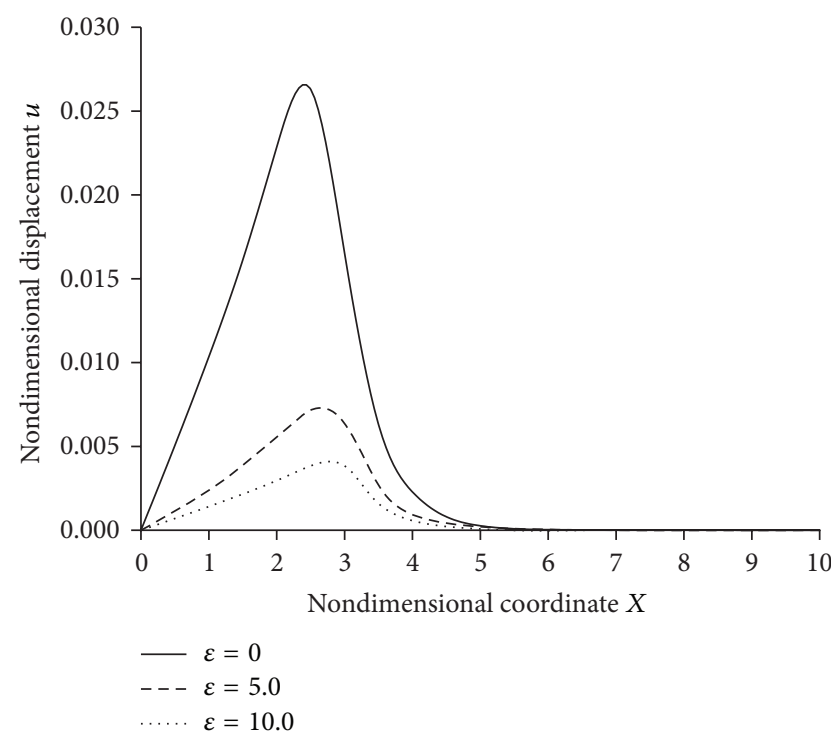

FIGURE 9: Nondimensional displacement distribution for different $\varepsilon$ values.

In Figure 7, the three lines overlap because the magnetic field has no effect on the temperature. As shown in Figure 8, the absolute value of stress increases as the $\varepsilon$ value increases prior to the point where the curves intersect; then, after the intersection, the absolute value of stress decreases as the applied magnetic field value increases. Figure 9 for Case 3 shows that nondimensional displacement decreases as the magnetic field increases. The largest displacement value is in the case of $\varepsilon=0$, namely, in the absence of the magnetic field. This indicates that the magnetic field acts to damp the thermal expansion deformation of the rod.

In Figures 10-12 (Case 4), the solid line, dash line, and dot line refer to $\vartheta=0.5, \vartheta=0.75$, and $\vartheta=1.0$, respectively. As shown in Figure 10, the temperature increases as the $\vartheta$ 


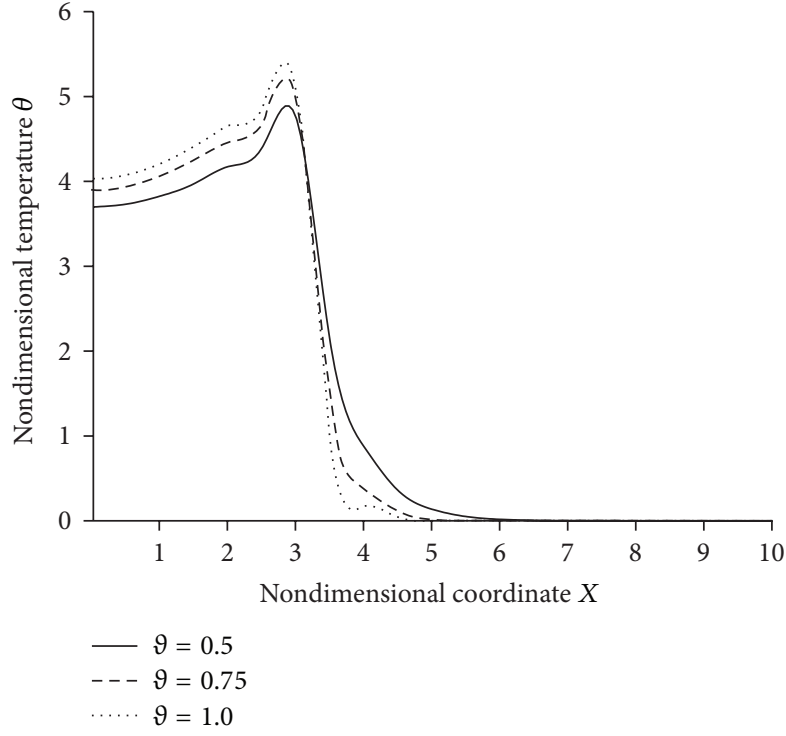

FIGURE 10: Nondimensional temperature distribution for different $\vartheta$ values.

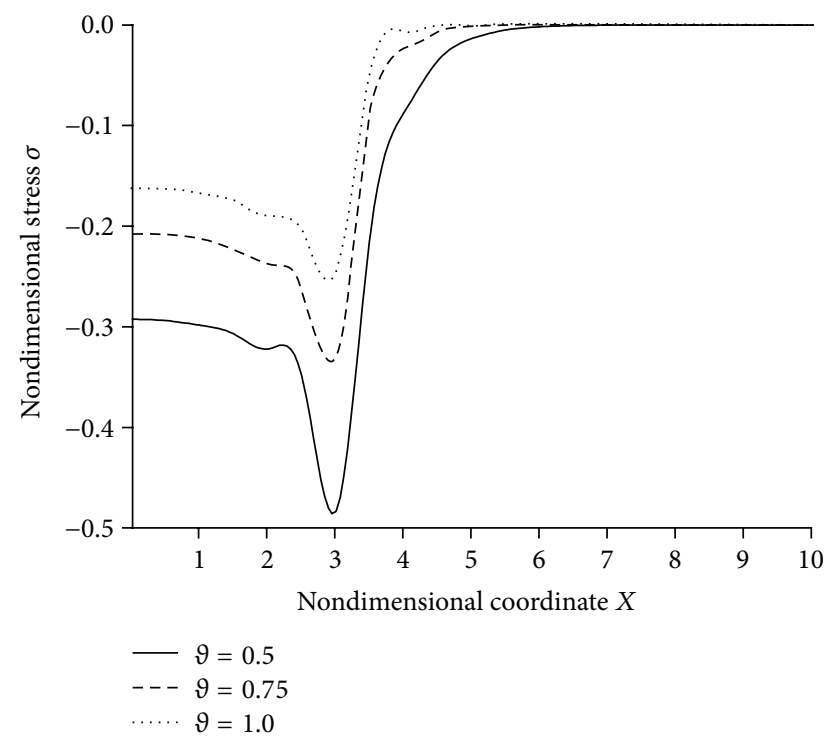

FIGURE 11: Nondimensional stress distribution for different $\vartheta$ values.

value increases before the three curves intersect, at which point it decreases as the temperature-dependent properties increase. Figures 11 and 12 show where the displacement and absolute value of stress decrease as the temperaturedependent properties increase.

Figures 13-15 (Case 5) show the variations of the nondimensional temperature, stress, and displacement, respectively, which demonstrate the effects of the fractional order parameter on the variations of the considered variables. To this end, a series of values of the fractional order parameter $\alpha$ within the region $(0,1]$ are tested through numerical calculation. As representation of the effect of $\alpha$, three typical values of $\alpha, \alpha=0.25, \alpha=0.5$, and $\alpha=1.0$, are considered. Figures 13-15 show that temperature, displacement, and

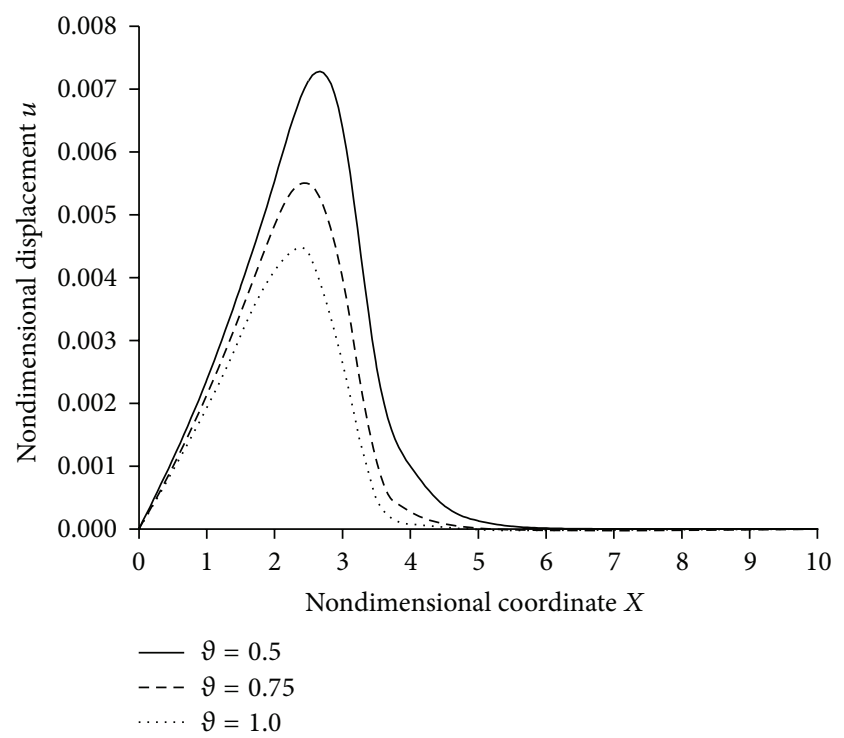

FIgURE 12: Nondimensional displacement distribution for different $\vartheta$ values.

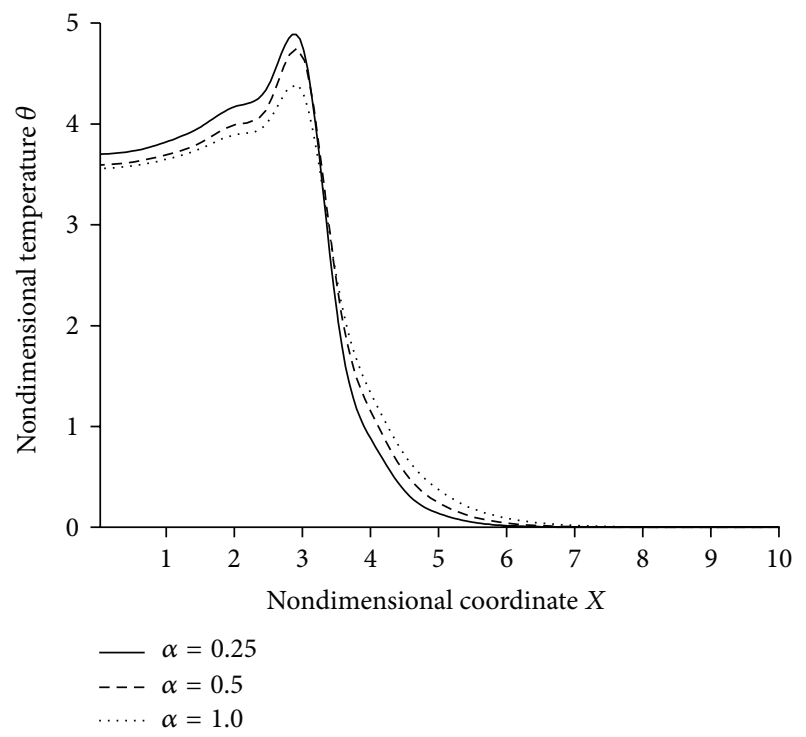

FIGURE 13: Nondimensional temperature distribution for different $\alpha$ values.

absolute stress value all decrease as the fractional order value increases prior to the intersection of the three curves. After the intersection, however, all considered variables increase with the increasing of the fractional order parameter.

\section{Conclusions}

A generalized magnetothermoelastic rod with temperaturedependent properties subjected to a moving heat source is investigated in the fractional order theory. The results provided the following most notable conclusions: 


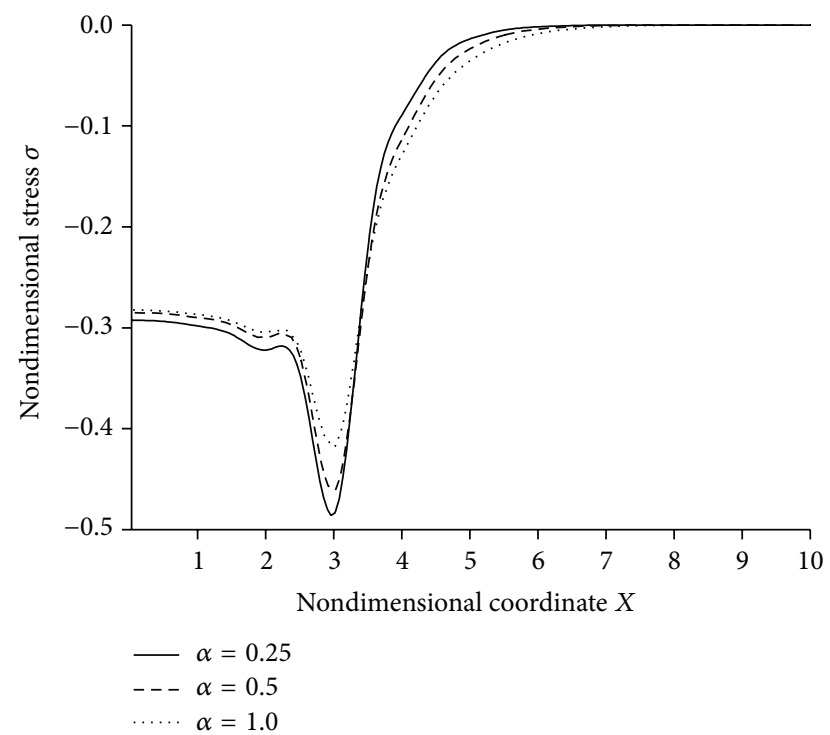

FIGURE 14: Nondimensional stress distribution for different $\alpha$ values.

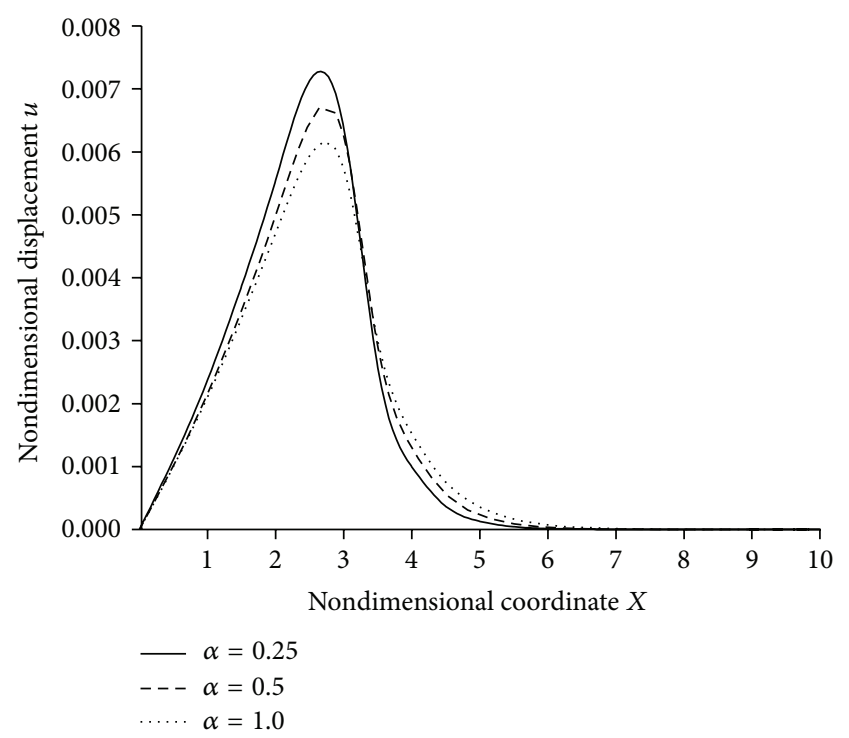

FIGURE 15: Nondimensional displacement distribution for different $\alpha$ values.

(1) The effects of the fractional parameter $\alpha$ on all the considered variables are very significant, as clearly evidenced by the peak values of the curves.

(2) The magnetic field also has a significant effect on the solution of the nondimensional displacement and stress but barely influences the variation in nondimensional temperature.

(3) The temperature-dependent properties play a significant role on all distributions.

As a final remark, the results presented in this paper should prove useful for researchers in material sciences, designers of new materials, low-temperature physics researchers, and those working to further develop the theory of thermoelasticity with fractional calculus. The introduction of fractional calculus and variable temperature-dependent modulus to the generalized thermoelastic rod may provide a more realistic model for these studies.

\section{Nomenclature}

J: Current density vector

$U: \quad$ Displacement vector

$B: \quad$ Magnetic induction vector

$\sigma_{i j}$ : The components of stress tensor

$e_{i j}$ : The components of strain tensor

$e_{k k}$ : Cubic dilation

$u_{i}$ : The components of displacement vector

$\theta: \quad \theta=T-T_{0}$

$T: \quad$ Absolute temperature of the medium

$T_{0}$ : Reference temperature

$\kappa_{i j}$ : The coefficient of thermal conductivity

$\tau_{0}$ : Thermal relaxation time

$\rho: \quad$ Mass density

$C_{E}$ : Specific heat at constant strain

$\lambda$, $\mu$ : Lame's constants

$\alpha_{t}:$ Linear thermal expansion coefficient

Q: The strength of the applied heat source per unit mass

$\gamma: \quad(3 \lambda+2 \mu) \alpha_{t}$

$\eta: \quad$ The entropy density

$q_{i}$ : The components of heat flux vector

$v$ : Velocity of the moving heat source

$\alpha$ : Constant parameter such that $0<\alpha \leq 1$.

\section{Competing Interests}

The authors declare that they have no competing interests.

\section{References}

[1] M. A. Biot, "Thermoelasticity and irreversible thermodynamics," Journal of Applied Physics, vol. 27, pp. 240-253, 1956.

[2] H. W. Lord and Y. Shulman, "A generalized dynamical theory of thermoelasticity," Journal of the Mechanics and Physics of Solids, vol. 15, no. 5, pp. 299-309, 1967.

[3] A. E. Green and K. A. Lindsay, “Thermoelasticity," Journal of Elasticity, vol. 2, no. 1, pp. 1-7, 1972.

[4] C. Catteneo, "A form of heat conduction equation which eliminates the paradox of instantaneous propagation," Compute Rendus, vol. 247, pp. 431-433, 1958.

[5] P. Vernotte, "Some possible complications in the phenomenon of thermal conduction," Compute Rendus, vol. 252, pp. 21902191, 1961.

[6] H. M. Youssef, "Generalized thermoelastic infinite medium with spherical cavity subjected to moving heat source," Computational Mathematics and Modeling, vol. 21, pp. 212-225, 2010.

[7] H. H. Sherief and N. M. El-Maghraby, "Effect of body forces on a 2D generalized thermoelastic long cylinder," Computers and Mathematics with Applications, vol. 66, no. 7, pp. 1181-1191, 2013.

[8] M. I. A. Othman and I. A. Abbas, "Effect of rotation on plane waves in generalized thermomicrostretch elastic solid: comparison of different theories using finite element method," Canadian Journal of Physics, vol. 92, no. 10, pp. 1269-1277, 2014. 
[9] H. H. Sherief and F. A. Hamza, "Modeling of variable thermal conductivity in a generalized thermoelastic infinitely long hollow cylinder," Meccanica, vol. 51, no. 3, pp. 551-558, 2016.

[10] Y. Heydarpour and M. Aghdam, "Transient analysis of rotating functionally graded truncated conical shells based on the LordShulman model," Thin-Walled Structures, vol. 104, pp. 168-184, 2016.

[11] Y. Wang, D. Liu, Q. Wang, and J. Zhou, "Asymptotic analysis of thermoelastic response in functionally graded thin plate subjected to a transient thermal shock," Composite Structures, vol. 139, pp. 233-242, 2016.

[12] T. He and L. Cao, "A problem of generalized magnetothermoelastic thin slim strip subjected to a moving heat source," Mathematical and Computer Modelling, vol. 49, no. 7-8, pp. 1710-1720, 2009.

[13] H. H. Sherief and S. E. Khader, "Propagation of discontinuities in electromagneto generalized thermoelasticity in cylindrical regions," Meccanica, vol. 48, no. 10, pp. 2511-2523, 2013.

[14] I. A. Abbas and A. M. Zenkour, "LS model on electro-magnetothermoelastic response of an infinite functionally graded cylinder," Composite Structures, vol. 96, pp. 89-96, 2013.

[15] N. Sarkar, "Generalized magneto-thermoelasticity with modified Ohm's law under three theories," Computational Mathematics and Modeling, vol. 25, no. 4, pp. 544-564, 2014.

[16] P. Pal, P. Das, and M. Kanoria, "Magneto-thermoelastic response in a functionally graded rotating medium due to a periodically varying heat source," Acta Mechanica, vol. 226, no. 7, pp. 2103-2120, 2015.

[17] M. C. Singh and N. Chakraborty, "Reflection of a plane magneto-thermoelastic wave at the boundary of a solid halfspace in presence of initial stress," Applied Mathematical Modelling, vol. 39, no. 5-6, pp. 1409-1421, 2015.

[18] S. M. Said, "Influence of gravity on generalized magnetothermoelastic medium for three-phase-lag model," Journal of Computational and Applied Mathematics, vol. 291, pp. 142-157, 2016.

[19] M. Caputo, "Vibrations on an infinite viscoelastic layer with a dissipative memory," Journal of the Acoustical Society of America, vol. 56, pp. 897-904, 1974.

[20] R. L. Bagley and P. J. Torvik, "A theoretical basis for the application of fractional calculus to viscoelasticity," Journal of Rheology, vol. 27, no. 3, pp. 201-210, 1983.

[21] R. C. Koeller, "Applications of fractional calculus to the theory of viscoelasticity," Journal of Applied Mechanics, vol. 51, no. 2, pp. 299-307, 1984.

[22] Y. A. Rossikhin and M. V. Shitikova, "Applications of fractional calculus to dynamic problems of linear and nonlinear hereditary mechanics of solids," Applied Mechanics Reviews, vol. 50, no. 1, pp. 15-67, 1997.

[23] Y. Z. Povstenko, "Fractional heat conduction equation and associated thermal stress," Journal of Thermal Stresses, vol. 28, no. 1, pp. 83-102, 2005.

[24] H. M. Youssef, "Theory of fractional order generalized thermoelasticity," Journal of Heat Transfer, vol. 132, no. 6, Article ID 061301, 7 pages, 2010.

[25] N. Sarkar and A. Lahiri, "Effect of fractional parameter on plane waves in a rotating elastic medium under fractional order generalized thermoelasticity," International Journal of Applied Mechanics, vol. 4, no. 3, Article ID 1250030, 2012.

[26] H. M. Youssef, "State-space approach to fractional order twotemperature generalized thermoelastic medium subjected to moving heat source," Mechanics of Advanced Materials and Structures, vol. 20, no. 1, pp. 47-60, 2013.

[27] Y. J. Yu, X. G. Tian, and T. J. Lu, "Fractional order generalized electro-magneto-thermo-elasticity," European Journal of Mechanics. A. Solids, vol. 42, pp. 188-202, 2013.

[28] H. M. Youssef and I. A. Abbas, "Fractional order generalized thermoelasticity with variable thermal conductivity," Journal of Vibroengineering, vol. 16, pp. 4077-4087, 2014.

[29] Y. Q. Song, J. T. Bai, Z. Zhao, and Y. F. Kang, "Study on the vibration of optically excited microcantilevers under fractionalorder thermoelastic theory," International Journal of Thermophysics, vol. 36, no. 4, pp. 733-746, 2015.

[30] I. A. Abbas and H. M. Youssef, "Two-dimensional fractional order generalized thermoelastic porous material," Latin American Journal of Solids and Structures, vol. 12, no. 7, pp. 1415-1431, 2015.

[31] H. H. Sherief, A. M. A. El-Sayed, and A. M. Abd El-Latief, "Fractional order theory of thermoelasticity," International Journal of Solids and Structures, vol. 47, no. 2, pp. 269-275, 2010.

[32] S. Kothari and S. Mukhopadhyay, "A problem on elastic half space under fractional order theory of thermoelasticity," Journal of Thermal Stresses, vol. 34, no. 7, pp. 724-739, 2011.

[33] H. Sherief and A. M. Abd El-Latief, "Effect of variable thermal conductivity on a half-space under the fractional order theory of thermoelasticity," International Journal of Mechanical Sciences, vol. 74, pp. 185-189, 2013.

[34] T. H. He and Y. Guo, "A one-dimensional thermoelastic problem due to a moving heat source under fractional order theory of thermoelasticity," Advances in Materials Science and Engineering, vol. 2014, Article ID 510205, 9 pages, 2014.

[35] H. H. Sherief and A. M. Abd El-Latief, "Application of fractional order theory of thermoelasticity to a $1 D$ problem for a halfspace," ZAMM-Zeitschrift für Angewandte Mathematik und Mechanik, vol. 94, no. 6, pp. 509-515, 2014.

[36] H. H. Sherief and A. M. Abd El-Latief, "Application of fractional order theory of thermoelasticity to a $2 \mathrm{D}$ problem for a halfspace," Applied Mathematics and Computation, vol. 248, pp. 584-592, 2014.

[37] I. A. Abbas, "Eigenvalue approach to fractional order generalized magneto-thermoelastic medium subjected to moving heat source," Journal of Magnetism and Magnetic Materials, vol. 377, pp. 452-459, 2015.

[38] H. H. Sherief and A. M. Abd El-Latief, "A one-dimensional fractional order thermoelastic problem for a spherical cavity," Mathematics and Mechanics of Solids, vol. 20, no. 5, pp. 512-521, 2015.

[39] Y. B. Ma and T. H. He, "Dynamic response of a generalized piezoelectric-thermoelastic problem under fractional order theory of thermoelasticity," Mechanics of Advanced Materials and Structures, vol. 23, no. 10, pp. 1173-1180, 2016.

[40] M. A. Ezzat, A. S. El-Karamany, and A. A. Samaan, "The dependence of the modulus of elasticity on reference temperature in generalized thermoelasticity with thermal relaxation," Applied Mathematics and Computation, vol. 147, no. 1, pp. 169-189, 2004.

[41] M. N. Allam, K. A. Elsibai, and A. E. Abouelregal, "Magnetothermoelasticity for an infinite body with a spherical cavity and variable material properties without energy dissipation," International Journal of Solids and Structures, vol. 47, no. 20, pp. 2631-2638, 2010. 
[42] Q.-L. Xiong and X.-G. Tian, “Transient magneto-thermoelastic response for a semi-infinite body with voids and variable material properties during thermal shock," International Journal of Applied Mechanics, vol. 3, no. 4, pp. 881-902, 2011.

[43] A. E. Abouelregal, "Fractional order generalized thermopiezoelectric semi-infinite medium with temperaturedependent properties subjected to a ramp-type heating," Journal of Thermal Stresses, vol. 34, no. 11, pp. 1139-1155, 2011.

[44] M. I. A. Othman, Y. D. Elmaklizi, and S. M. Said, "Generalized thermoelastic medium with temperature-dependent properties for different theories under the effect of gravity field," International Journal of Thermophysics, vol. 34, no. 3, pp. 521-537, 2013.

[45] P. Pal, A. Kar, and M. Kanoria, "Fractional order generalised thermoelasticity to an infinite body with a cylindrical cavity and variable material properties," European Journal of Computational Mechanics, vol. 23, no. 1-2, pp. 96-111, 2014.

[46] Q. Xiong and X. Tian, "Generalized magneto-thermomicrostretch response of a half-space with temperaturedependent properties during thermal shock," Latin American Journal of Solids and Structures, vol. 12, no. 13, pp. 2562-2580, 2015.

[47] Y. Z. Wang, D. Liu, Q. Wang, and J. Z. Zhou, "Fractional order theory of thermoelasticity for elastic medium with variable material properties," Journal of Thermal Stresses, vol. 38, no. 6, pp. 665-676, 2015.

[48] G. Honig and U. Hirdes, "A method for the numerical inversion of Laplace transforms," Journal of Computational and Applied Mathematics, vol. 10, no. 1, pp. 113-132, 1984. 

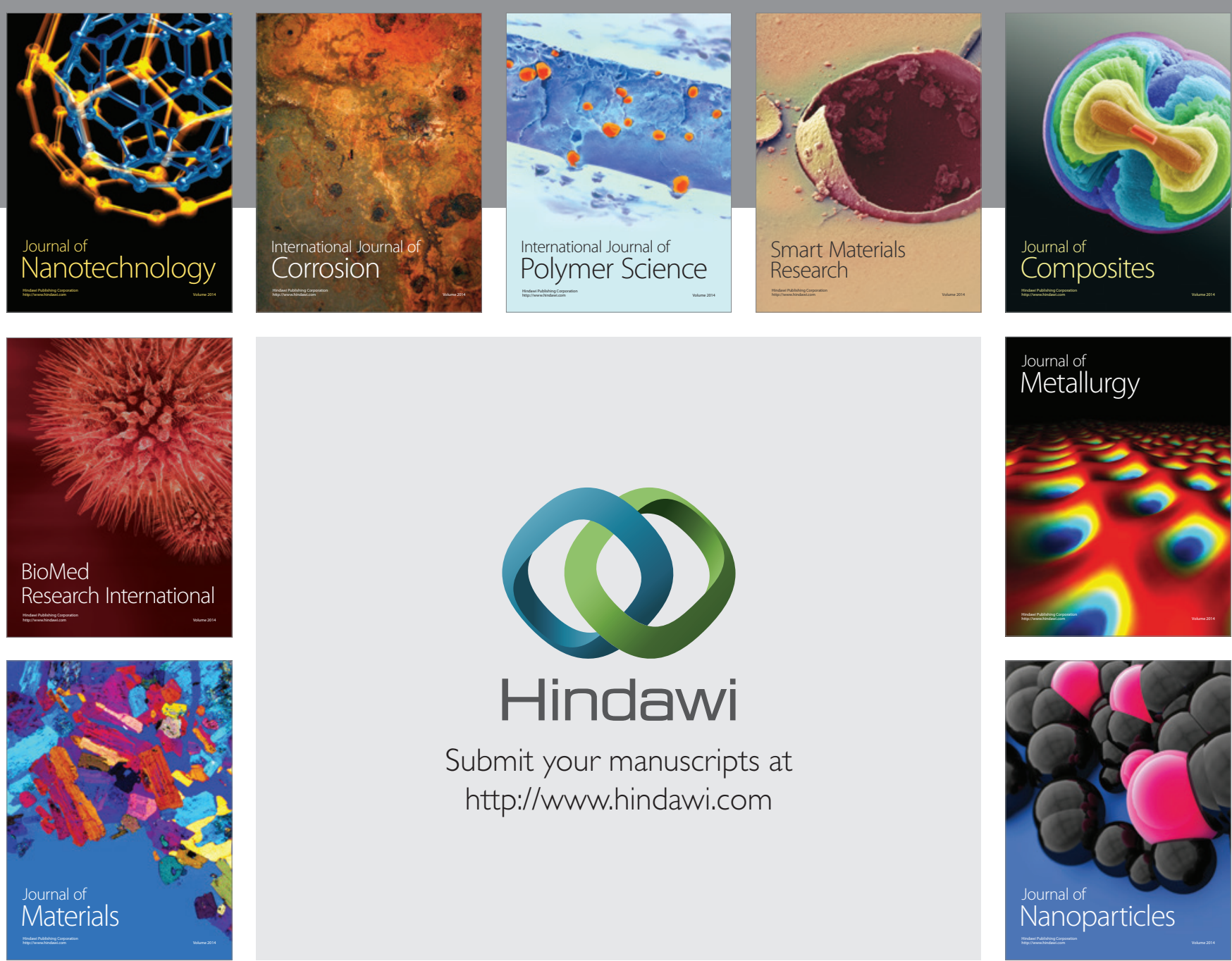

\section{Hindawi}

Submit your manuscripts at

http://www.hindawi.com

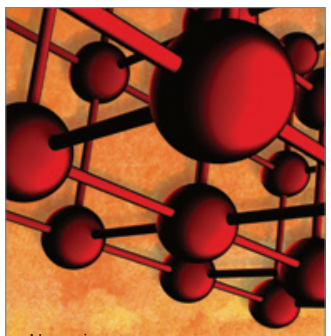

Materials Science and Engineering
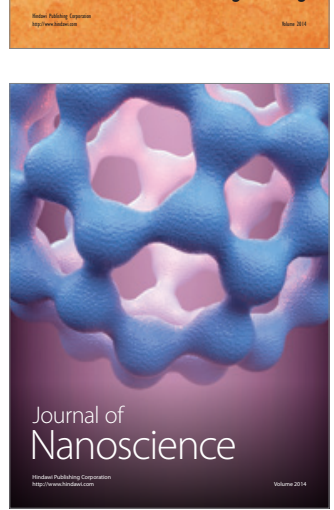
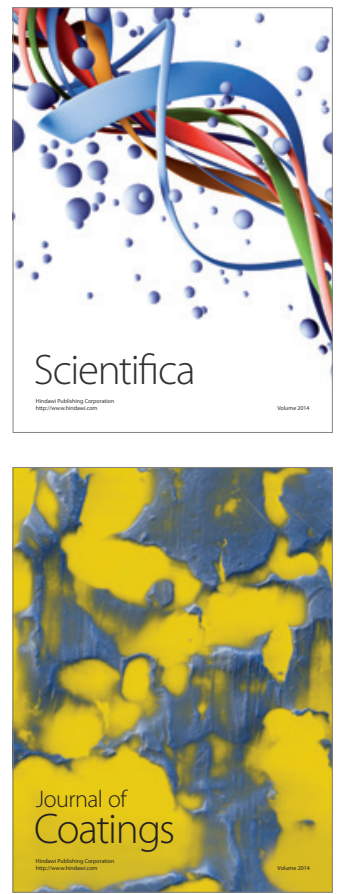
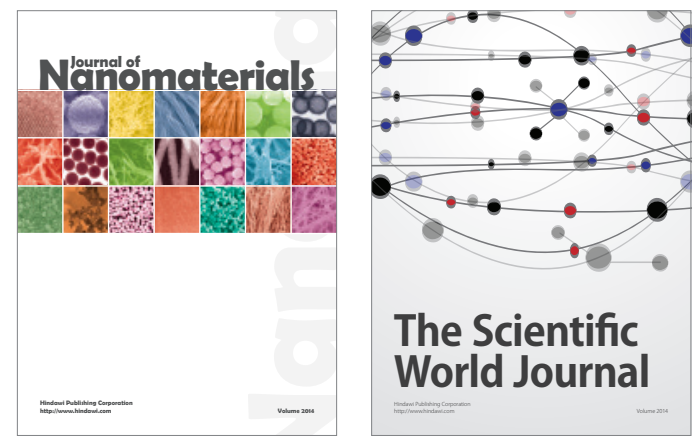

The Scientific World Journal
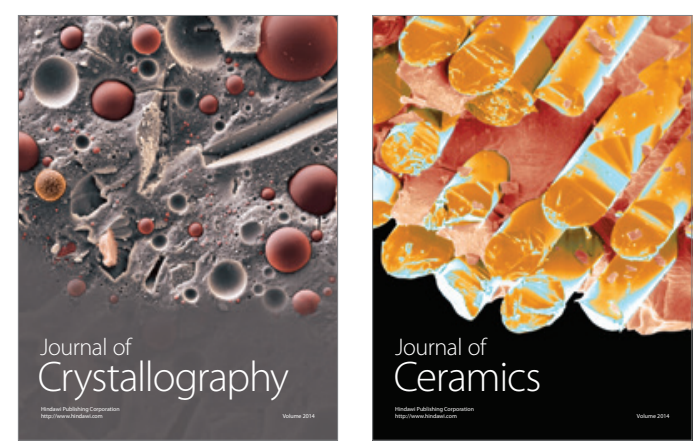
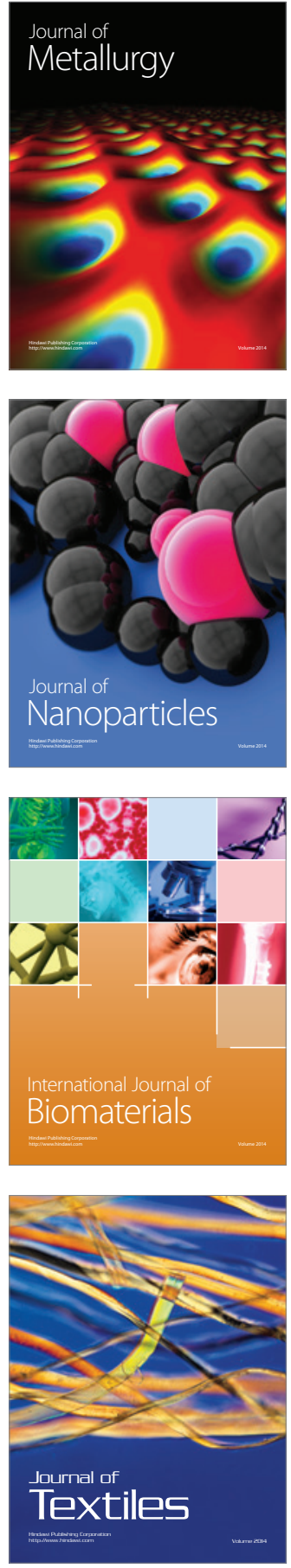